\title{
Severe Symptomatic Bradycardia After Intravenous Immunoglobulin Infusion: A Rare Manifestation
}

\author{
Steven Douedi' ${ }^{1}$, Abbas Alshami', Gina Francisco Ashforth', Obiora Maludum², Michael P. Carson' \\ 'Department of Medicine, Jersey Shore University Medical Center, New Jersey, United States \\ ${ }^{2}$ Department of Cardiology, Jersey Shore University Medical Center, New Jersey, United States
}

\section{Article Info}

\section{Article Notes}

Received: January 12, 2020

Accepted: February 3, 2020

\section{${ }^{*}$ Correspondence:}

${ }^{*}$ Dr. Steven Douedi, MD, Internal Medicine Residency Program, Department of Medicine, Jersey Shore University Medical Center, Hackensack Meridian Health, Neptune, NJ 07753 United States; Email: Steven.Douedi@hackensackmeridian.org.

(c) 2020 Douedi S. This article is distributed under the terms of the Creative Commons Attribution 4.0 International License.
Abstract

Intravenous immunoglobulins (IVIGs) are immunomodulating agents prepared using pooled plasma from thousands of human donors. These IVIGs have been used to treat a wide range of autoimmune, infectious, and idiopathic diseases. Their use in idiopathic thrombocytopenic purpura (ITP) was first described in 1981 and was found to be an effective alternative to splenectomy. The standard dose of IVIG in patients with ITP is 400 milligrams per kilogram body weight $(\mathrm{mg} / \mathrm{kg}$ ) daily for 5 days however recent data has shown a dose of $1 \mathrm{gram} / \mathrm{kilogram} /$ day for 2 days may be more effective. Side-effects during IVIG infusions have been reported in about 5 to $15 \%$ of patients. Cardiac related side-effects such as arrythmias, hypotension, and even myocardial infarction, being rare, have also been documented but are usually seen in patients with underlying cardiac pathologies. This article presents a 61-year-old male with no history of cardiac disease or arrhythmias who developed symptomatic bradycardia thirty minutes after intravenous immunoglobin infusion requiring multiple atropine injections and dopamine infusion over a 7-day hospitalization. The bradycardia resolved afterwards, and cardiac workup did not identify any underlying pathology.

Keywords: Immunoglobin; Bradycardia; Side effect; Cardiac; Dysrhythmia; Idiopathic thrombocytopenic purpura.

\section{Learning Objectives}

1. Increase healthcare providers' awareness of this serious cardiac side effect of intravenous immunoglobin (IVIG) infusion.

2. Highlight the need for recommendations on the readministrability of IVIG in patients who develop such side effect.

\section{Introduction}

Intravenous Immunoglobulins (IVIGs) are blood products pooled from a large number, usually 1,000 or more, of healthy donors ${ }^{1}$. Their use has recently increased as they showed efficacy in the management of a large variety of autoimmune, infectious, and hematologic diseases ${ }^{2}$. Several cardiac side effects due to IVIGs infusion have been reported including supraventricular tachycardia, hypotension, and bradycardia; however, these are usually rare and associated with an underlying heart disease ${ }^{3}$. We report a patient with no past cardiac history who developed symptomatic bradycardia after IVIGs infusion.

\section{Case Presentation}

A 61-year-old male with no medical issues was directed to the emergency department (ED) after his platelet (PLT) count was 
found to be 5000 cell/mm3 during routine blood work. He denied bruising, epistaxis, or gingival bleeding. Vital signs on admission were all within normal limits and the patients recorded weight was $97.9 \mathrm{~kg}$. Physical examination showed no lymphadenopathy, hepatosplenomegaly, petechiae, or ecchymoses. Repeat PLT count on admission to the hospital was 3000 cells $/ \mathrm{mm} 3$. Other laboratory results showed WBC 9.1 K/uL, Hgb 13.1 gm/dL, MCV 94.3 fL, RDW $14 \%$, immature platelet fraction $21.9 \%$. Electrocardiogram (ECG) on admission in the ED showed normal sinus rhythm with a heart rate in the $90 \mathrm{~s}$ beats per minute (bpm) (Figure 1). He was admitted with a presumptive diagnosis of idiopathic thrombocytopenic purpura (ITP). Treatment was started with two days of prednisone 50 milligrams (mg) twice daily but had no effect. He was then started on 3 days of dexamethasone $40 \mathrm{mg}$ intravenously (IV) with still no improvement, so $1 \mathrm{gm} / \mathrm{kg}$ (gram/kilogram) of intravenous immunoglobulin was infused at an initial rate of $5.88 \mathrm{gm} / \mathrm{hr}$. Thirty minutes after starting the dose (Gamunex-C 10\%, Grifols Therapeutics Inc, Barcelona) he developed chest pain and shortness of breath with heart rate $31 \mathrm{bpm}$, temperature 98.4 degrees Fahrenheit, respiratory rate 17 per $\min$, and blood pressure 109/64 mmHg. ECG within 30 minutes after IVIG showed sinus bradycardia with a heart rate of 30 s (Figure 2). Cardiac troponin levels $\mathrm{x} 2$ were obtained and were negative $(<0.04$

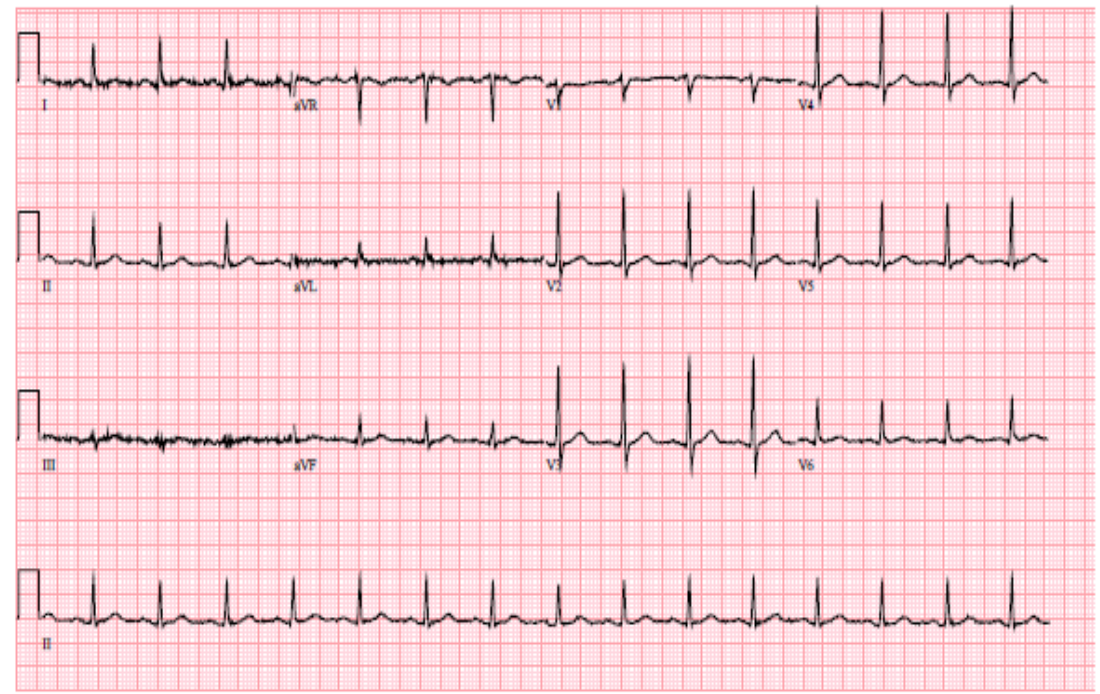

Figure 1. EKG on admission prior to IVIG infusion showing normal sinus rhythm with a heart rate of $92 \mathrm{bpm}$.

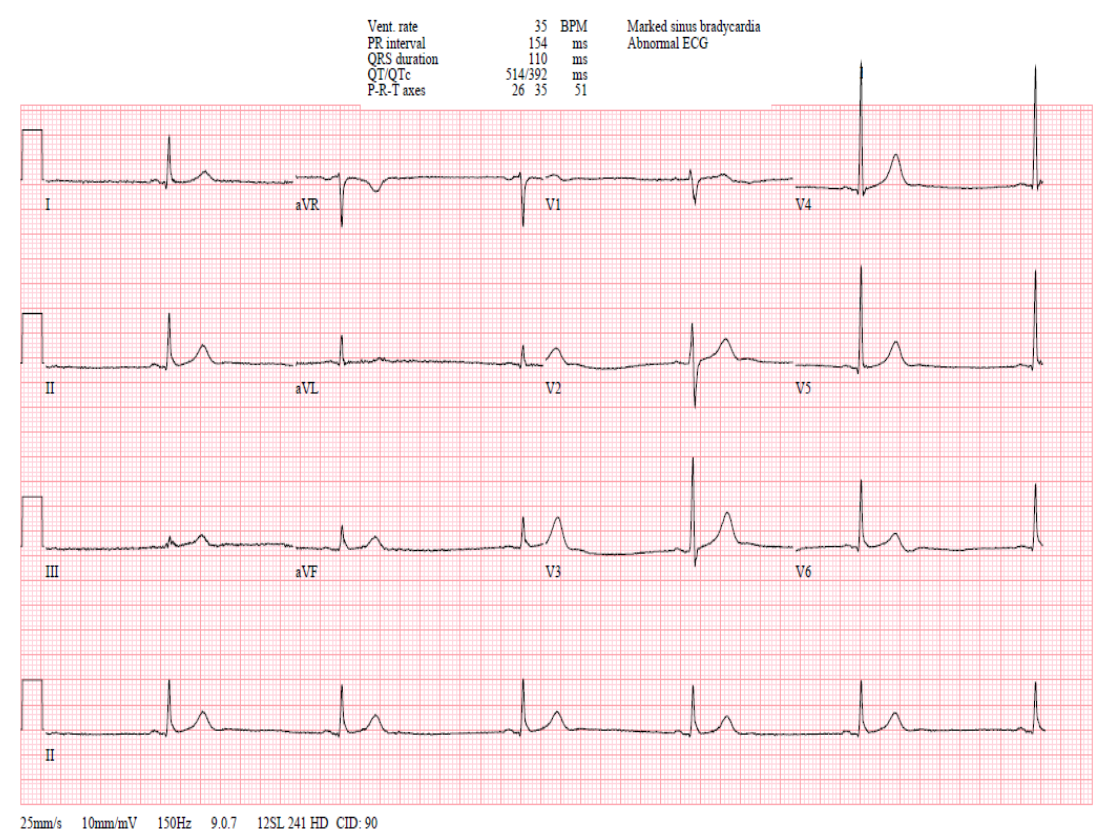

Figure 2: Electrocardiogram showing sinus bradycardia after the development of chest pain and shortness of breath 30 minutes after IVIG infusion. 


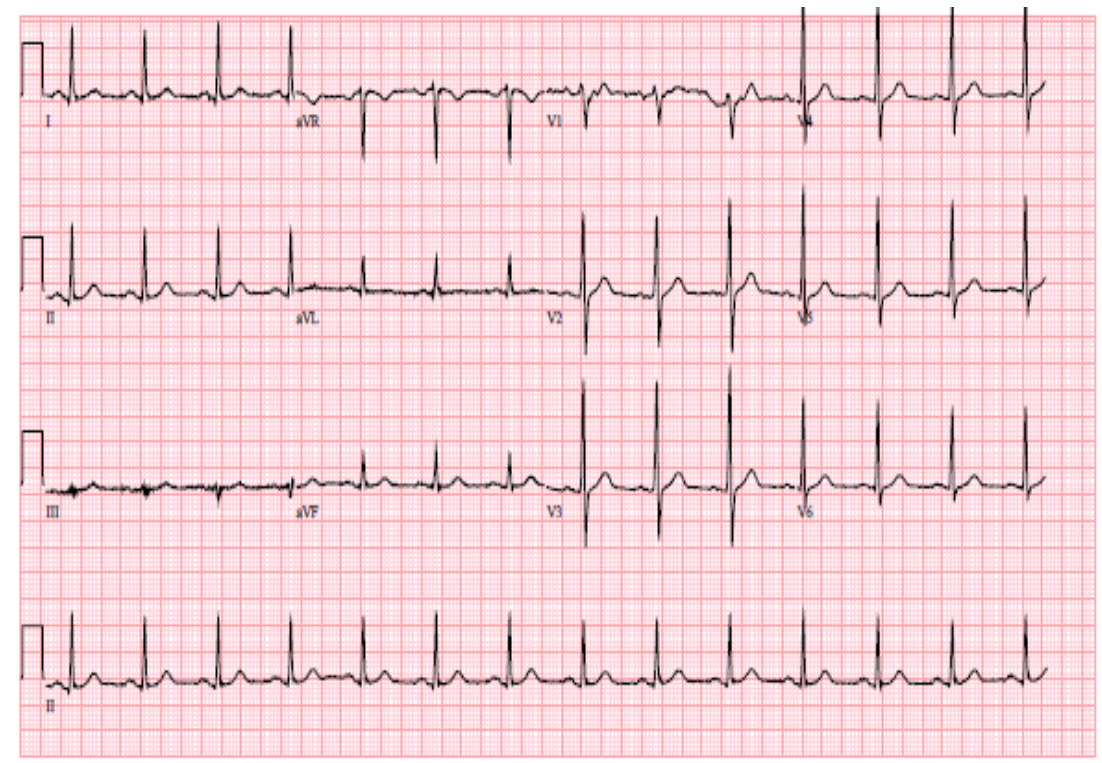

Figure 3. EKG after treatment with atropine and dopamine infusion showing normal sinus rhythm with a heart rate of $82 \mathrm{bpm}$.

$\mathrm{ng} / \mathrm{mL}$ ). Emergent transthoracic echocardiogram (TTE) was performed and showed an ejection fraction of $51-54 \%$ and no regional or global wall motion abnormalities. IV atropine $0.5 \mathrm{mg}$ corrected his heart rate to $60-70 \mathrm{bpm}$ with resolution of his symptoms. Six hours later he required another dose of atropine for a HR less than 35 . The patient required additional atropine $0.5 \mathrm{mg}$ doses 3-4 times a day and eventual dopamine infusion for 1 week. The patient's bradycardia resolved after treatment with atropine and dopamine (Figure 3) and due to adverse reactions, his ITP was ultimately treated with splenectomy rather than IVIG infusions.

\section{Discussion}

This case of prolonged symptomatic bradycardia stands in contrast to the majority of reported IVIGinduced dysrhythmia cases as most were in patients with underlying heart disease and/or a history of tachyarrhythmias. Savaşan and co-investigators reported 2 cases of supraventricular tachycardia (SVT) and accelerated ventricular rhythm during IVIG infusion in patients with underlying dysrhythmias before treatment with IVIG Similarly, Tufekci et al. reported the occurrence of SVT during IVIG infusion in two neonates with history of SVT ${ }^{5}$. This is, to our knowledge, only the 2nd case of IVIG induced symptomatic bradycardia in a person without underlying heart disease. The first case reported was by Raheja and coinvestigators who reported in a letter to the editor a similar case of ITP who received IVIG and developed 4 episodes of asymptomatic sinus bradycardia following sequential IVIG infusions ${ }^{6}$. Moreover, the magnitude of the symptomatic bradycardia in the aforementioned case correlated with the dose of the administered IVIG ${ }^{6}$. The mechanism by which IVIG causes cardiovascular side effects remains unclear.
Our patient did not have any underlying cardiac history that might predispose him to bradycardia, and the unremarkable TTE and troponins after the episode excluded any structural heart disease that can explain it. Although our patient received corticosteroids, which have been reported to cause bradycardia ${ }^{7-9}$, the temporal association between the IVIG infusion and the development of bradycardia suggest the IVIG as etiology. In addition, the corticosteroids were given for a total of 5 days without any bradycardic episodes or acute side effects prior to receiving his first IVIG infusion.

The risk versus benefit of discontinuation of future IVIG infusion in patients who develop cardiac side effects remain to be determined by larger clinical studies. Due to the prolonged effect in our patient, IVIG was deemed contraindicated, and his ITP was ultimately treated with splenectomy with good outcomes.

\section{Conclusion}

Intravenous Immunoglobin infusion can result in severe bradycardia, even in patients with no previous cardiac history. Albeit rare, it is imperative that physicians recognize, diagnose and treat the rare occurrence of symptomatic bradycardia after IVIG infusion to rapidly and appropriately manage patients and prevent morbidity and mortality from severe bradycardia induced by IVIG infusions.

\section{Conflicts of Interest}

The authors have no conflicts of interest to declare

\section{Acknowledgements}

Not applicable 


\section{References}

1. Barahona Afonso AF, João CMP. The Production Processes and Biological Effects of Intravenous Immunoglobulin. Biomolecules. 2016; 6(1): 15.

2. Kaveri SV, Dietrich G, Hurez V, et al. Intravenous immunoglobulins (IVIg) in the treatment of autoimmune diseases. Clin Exp Immunol. 1991; 86(2): 192-8

3. Guo Y, Tian X, Wang X, et al. Adverse Effects of Immunoglobulin Therapy. Front Immunol. 2018; 9: 1299.

4. Savaan S, Tuzcu V, Warrier I, et al. Cardiac Rhythm Abnormalities During Intravenous Immunoglobulin G Infusion for Treatment of Thrombocytopenia. J Pediatr Hematol Oncol. 1997; 19(3): 254-7.

5. Tufekci S, Coban A, Bor M, et al. Cardiac rhythm abnormalities during intravenous immunoglobulin G(IVIG) infusion in two newborn infants: coincidence or association? Clin case reports. 2015; 3(9): 731-4.

6. Raheja H, Kumar V, Hollander G, et al. Intravenous ImmunoglobulinInduced Profound Bradycardia in a Patient With Idiopathic Thrombocytopenic Purpura. Am J Ther. 2018; 25(5): e572-4.

7. Stroeder J, Evans C, Mansell H. Corticosteroid-induced bradycardia: Case report and review of the literature. Can Pharm J Ott. 2015; 148(5): 235-40.

8. Tvede N, Nielsen LP, Andersen V. Bradycardia After High-Dose Intravenous Methylprednisolone Therapy. Scand J Rheumatol. 1986; 15(3): 302-4

9. Taylor MR, Gaco D. Symptomatic Sinus Bradycardia After a Treatment Course Of High-dose Oral Prednisone. J Emerg Med. 2013; 45(3): e55-8. 